\title{
A Causal Role for Brain-Derived Neurotrophic Factor in the Homeostatic Regulation of Sleep
}

\author{
Ugo Faraguna, ${ }^{1,2}$ Vladyslav V. Vyazovskiy, ${ }^{1}$ Aaron B. Nelson, ${ }^{1}$ Giulio Tononi, ${ }^{1}$ and Chiara Cirelli ${ }^{1}$ \\ ${ }^{1}$ Department of Psychiatry, University of Wisconsin-Madison, Madison, Wisconsin 53719, and 2Scuola Superiore Sant'Anna, 56127 Pisa, Italy
}

\begin{abstract}
Slow-wave activity (SWA), the EEG power between 0.5 and $4 \mathrm{~Hz}$ during non-rapid eye movement (NREM) sleep, is one of the best characterized markers of sleep need, because it increases as a function of preceding waking duration and decreases during sleep, but the underlying mechanisms remain unknown. We hypothesized that SWA is high at sleep onset because it reflects the occurrence, during the previous waking period, of widespread synaptic potentiation in cortical and subcortical areas. Consistent with this hypothesis, we recently showed that the more rats explore, the stronger is the cortical expression of BDNF during wakefulness, and the larger is the increase in SWA during the subsequent sleep period. There is compelling evidence that BDNF plays a causal role in synaptic potentiation, and exogenous application of BDNF in vivo is sufficient to induce long-term increases in synaptic strength. We therefore performed cortical unilateral microinjections of BDNF in awake rats and measured SWA during the subsequent sleep period. SWA during NREM sleep was higher in the injected hemisphere relative to the contralateral one. The effect was reversible within $2 \mathrm{~h}$, and did not occur during wakefulness or rapid eye movement sleep. Asymmetries in NREM SWA did not occur after vehicle injections. Furthermore, microinjections, during wakefulness, of a polyclonal anti-BDNF antibody or K252a, an inhibitor of BDNF TrkB receptors, led to a local SWA decrease during the following sleep period. These effects were also reversible and specific for NREM sleep. These results show a causal link between BDNF expression during wakefulness and subsequent sleep regulation.
\end{abstract}

Key words: sleep homeostasis; cerebral cortex; EEG; rat; BDNF; synaptic plasticity

\section{Introduction}

Sleep is homeostatically regulated, in that its duration and intensity increase with the duration of the preceding waking period (Borbely, 1982). The best established electrophysiological correlate of sleep intensity is slow-wave activity (SWA), the EEG power between 0.5 and $4.0 \mathrm{~Hz}$ during non-rapid eye movement (NREM) sleep. In mammals and birds, SWA increases as a function of the duration of wakefulness and decreases during sleep (Achermann and Borbely, 2003; Martinez-Gonzalez et al., 2008). SWA is further increased by sleep deprivation and reduced by naps. Although SWA and sleep duration often change together, SWA can also increase significantly without major increases in sleep duration (Tobler et al., 1994; Tobler, 2005). Thus, SWA represents a sensitive marker of sleep pressure and sleep need. However, the biological process responsible for the increase of SWA as a function of previous wakefulness remains unknown.

Although sleep is a global phenomenon, increasing evidence suggests that SWA may be regulated locally by use-dependent processes, especially those linked to synaptic plasticity. In humans, SWA was locally increased over a parietal cortical area

Received Dec. 13, 2007; revised March 6, 2008; accepted March 6, 2008.

This work was supported by the National Institute of Mental Health Grant P20 MH077967 (project l; principal investigator, C.C.). We thank Danielle Ries, Christopher L. Douglas, and Jonathan Highness for excellent technical assistance.

Correspondence should be addressed to Dr. Chiara Cirelli, Department of Psychiatry, University of WisconsinMadison, 6001 Research Park Boulevard, Madison, WI 53719. E-mail: ccirelli@wisc.edu.

DOI:10.1523/JNEUROSCI.5510-07.2008

Copyright $\odot 2008$ Society for Neuroscience $\quad$ 0270-6474/08/284088-08\$15.00/0 involved in learning a visuomotor task, compared with a kinematically identical control task not requiring learning (Huber et al., 2004). Conversely, arm immobilization during the day led to decreased motor performance and sensory evoked responses, consistent with synaptic depression, and was followed by reduced SWA over contralateral sensorimotor cortex (Huber et al., 2006). Computer simulations indicate that stronger synapses lead to increased SWA by enhancing neuronal synchronization, whereas weaker synapses have the opposite effect (Esser et al., 2007).

A recent study in rats has provided molecular evidence for a possible link between neuronal plasticity and SWA regulation (Huber et al., 2007a). The study found that the more rats explored during waking, the larger was the cortical induction of plasticity-related genes BDNF, Arc, Homer, and NGFI-A. Crucially, although all rats had been awake for the same period of time, those exploring the most had the highest SWA during subsequent sleep. Moreover, waking exploratory behavior and SWA response were positively correlated, and both were positively correlated with cortical BDNF expression. These correlative results raise the question whether BDNF may play a causal role in mediating the link between neuronal plasticity and SWA regulation.

BDNF, a member of the neutrophin family (Thoenen, 2000), binds to two membrane receptors, the p75 receptor and the tyrosine kinase receptor TrkB, but all its synaptic effects have been attributed to TrkB (Nagappan and Lu, 2005). The expression and secretion of BDNF are strongly activity dependent, and its role in synaptic potentiation is well established (for review, see Lu, 2003). BDNF is needed in the extracellular space within minutes 
of a burst stimulation to obtain long-term synaptic potentiation (LTP), suggesting that it may have a crucial role in LTP induction (Kossel et al., 2001). Moreover, in vivo infusions of BDNF are sufficient to directly induce hippocampal LTP (Messaoudi et al., 1998, 2002, 2007). Blocking BDNF, however, impairs LTP induction and maintenance (Akaneya et al., 1997) and promotes longterm depression in the visual cortex (Jiang et al., 2003).

If BDNF plays a causal role in regulating SWA, manipulations that increase or decrease cortical BDNF levels during waking should result in an increased or decreased sleep SWA. Here, to test this prediction, we performed intracortical microinjections of either BDNF or BDNF blockers while the rats were awake, and measured the following SWA response.

\section{Materials and Methods}

Animals, surgery, and recordings. Male WKY rats (Charles River Laboratories, Wilmington, MA; 250-300 g at time of surgery) were maintained on a $12 \mathrm{~h}$ light/dark cycle (lights on at 10:00 A.M.; room temperature, $23 \pm 1{ }^{\circ} \mathrm{C}$ ). Under deep isoflurane anesthesia (1.5-2\% volume), rats were chronically implanted for chronic polysomnographic recordings in the frontal cortex ( $\mathrm{B},+2-3 \mathrm{~mm} ; \mathrm{L}, 2-3 \mathrm{~mm})$ using bipolar local field potential (LFP) cannula/bipolar wire electrodes $(0.2 \mathrm{~mm}$ stainless-steel wire electrodes; 26 ga stainless-steel guide cannula; Simultaneous Bipolar Electrode-Microdialysis System; PlasticsOne, Roanoke, VA). The LFP bipolar wire electrodes had $1 \mathrm{~mm}$ vertical and $0.8 \mathrm{~mm}$ horizontal separation between electrode tips. The shallow lead was aimed at cortical layers I-II. Electrodes were fixed to the skull with dental cement. Two stainless-steel wires (diameter, $0.4 \mathrm{~mm}$ ) inserted into the neck muscles were used to record the electromyogram (EMG). Immediately after surgery, the animals were individually placed in transparent Plexiglas cages $(36.5 \times 25 \times 46 \mathrm{~cm})$, and kept in sound-attenuating recording boxes for the duration of the experiment. At least $8 \mathrm{~d}$ were allowed for recovery after surgery, and experiments were started only after the sleep/waking cycle had fully normalized. The rats were connected by means of a flexible cable to a commutator (Airflyte, Bayonne, NJ) and recorded continuously. To habituate the animals to the sleep deprivation procedure (see below), every day beginning the day after surgery rats were handled and exposed to a novel object between 10:00 and 10:30 A.M. Video recordings were performed continuously with infrared cameras (OptiView Technologies, Potomac Falls, VA) and stored in real-time (AVerMedia Technologies, Milpitas, CA). To verify that the animals were fully entrained to the light/dark cycle, all cages were equipped with Chronokit activity monitor infrared sensors (Stanford Chronokit; Stanford Software Systems, Santa Cruz, CA). Animal protocols followed the National Institutes of Health Guide for the Care and Use of Laboratory Animals and were in accordance with institutional guidelines.

Data acquisition. Rats were connected by means of a flexible cable and a commutator to a Grass mod. 8 polygraph (Grass Instruments, West Warick, RI). LFP and EMG signals were conditioned by analog filters (high pass, $0.1 \mathrm{~Hz}$; low pass, $35 \mathrm{~Hz}$ ), digitalized at $128 \mathrm{~Hz}$ (Kissei America, Irvine, CA) and stored on a computer. Waking, NREM, and rapid eye movement (REM) sleep states were visually determined in $4 \mathrm{~s}$ epochs based on the LFP, electrocorticogram (obtained by referencing the superficial lead of the LFP electrode to a "neutral" cerebellar screw), and EMG recordings according to standard criteria. Visual scoring was manually performed off-line (Sleep Sign; Kissei COMTEC, Matsumoto, Japan). Artifacts were always removed simultaneously from all derivations and vigilance states could always be determined. LFP power spectra (fast Fourier transform routine, Hanning window) were calculated for consecutive $4 \mathrm{~s}$ epochs within the $0.25-20.0 \mathrm{~Hz}$ frequency range. Sleep latency was defined as the time between the start of the injection and the first consolidated sleep episode (sleep episode lasting for at least $1 \mathrm{~min}$, interrupted by no more than $4 \mathrm{~s}$ of wakefulness).

Sleep deprivation. Total sleep deprivation began at light onset and was performed for $4 \mathrm{~h}$ by exposing the animals to a variety of novel objects, by transferring bedding material between cages, and by tapping on the cage whenever the rats appeared inactive and slow waves became evident on real-time EEG monitoring. Rats were never disturbed when they were spontaneously awake, feeding or drinking. This sleep deprivation method was used in all rats, was the same used in our previous study (Huber et al., 2007a), and was selected because this procedure enhances exploratory behavior and induces widespread plasticity in cortical and hippocampal circuits [Huber et al. (2007a), their references]. The EEG analysis focused on the frontal cortex because this brain region shows the most consistent changes in sleep SWA after both spontaneous and forced wakefulness (Vyazovskiy et al., 2004). The underlying assumption, based on our previous findings (Huber et al., 2007a), was that the exposure to novelty and the resulting exploratory behavior, rather than simply the lack of sleep, are the primary factors responsible for inducing changes in sleep SWA.

Injection procedure. The animals were habituated to the injection procedure by daily handling. On the injection day, the procedure included several steps: removal of the obturator, insertion of the injector, and delivery of the compound. Total volume and infusion rate were established in pilot experiments to allow the injected drug to affect the LFP signal only locally. Specifically, we confirmed that the diffusion of the dye Pontamine blue ( $2 \%$ ) was confined to the frontal area surrounding the electrode. Also, the injection of a solution of $20 \%$ lidocaine, a sodium channel blocker that causes a temporary inhibition of neuronal activity (Amzica and Steriade, 1995; Jha et al., 2005), suppressed EEG activity only at the corresponding (frontal) LFP, and did not affect either the contralateral frontal LFP, or the parietal LFPs. All solutions were infused through polyethylene tubing ( $5 \mu \mathrm{l} /$ injection delivered over $10 \mathrm{~min}$ ) connected to a Hamilton syringe controlled by a microinfusion pump (CMA 400 Syringe Pump; CMA Microdialysis, Solna, Sweden). The injector was removed 5-10 min after the end of the injection to avoid backflows and immediately replaced with the obturator. In each animal, only one side was injected (same side throughout the entire experiment).

All BDNF (or BDNF vehicle) injections were performed soon after light onset (between 10:22 and 10:49 A.M.). After the injection, rats were kept awake for $1 \mathrm{~h}$ by exposure to novel objects. Each animal received two injections in the right ( $n=2$ rats) or left ( $n=5$ rats) frontal cortex, one of vehicle and one of BDNF, according to a counterbalanced design (BDNF was injected first in three cases, and vehicle in four cases). Injections were at least $4 \mathrm{~d}$ apart. Human recombinant BDNF (Alomone Labs, Jerusalem, Israel) was diluted in PBS (150 mM NaCl, $10 \mathrm{~mm}$ phosphate buffer, $\mathrm{pH} 7.0$, and $0.004 \%$ Tween 20 ) to a final concentration of $1 \mu \mathrm{g} / \mu \mathrm{l}$. A similar dose was perfused in vivo in the hippocampus to induce LTP (Akaneya et al., 1997; Messaoudi et al., 1998, 2002) and was used in cortical slices to potentiate field potential and EPSCs (Akaneya et al., 1997).

The injections of BDNF blockers [methyl-9- $(S)-12(R)$-epoxy-1 H-diindolo[1,2,3-fg: $\left.\quad 32^{\prime} 1^{\prime}-\mathrm{kl}\right]$ pyrrolo[3,4-i][1,6]benzodiazocine-2,3,9,10,11,12hexahydro-10- $(R)$-hydroxy-9-methyl-1-oxo-10-carboxilate (K252a) and anti-BDNF antibody, and their corresponding controls] were performed between 10:15 and 10:42 A.M. As for BDNF, each rat received two injections, spaced at least $4 \mathrm{~d}$ apart, either of K252a and its vehicle, or of anti-BDNF and anti-NT3 (used as control for anti-BDNF). K252a was injected in six rats (three on each side of the frontal cortex), and anti-BDNF antibody in seven rats (four on the right frontal cortex). After the injection, the animals were kept awake for $\sim 4 \mathrm{~h}$ (until 2:00 P.M.) by exposure to novel objects. AntiBDNF (Promega, Madison, WI) was injected at the same concentrations (0.5 $\mathrm{mg} / \mathrm{ml}$ ) previously shown to have function-blocking effects in vivo (Caleo et al., 2000). Anti-NT3 (Promega) was injected at the same concentration (0.5 $\mathrm{mg} / \mathrm{ml}$ ). K252a (Calbiochem, La Jolla, CA) was injected at $40 \mu \mathrm{m}$ in $0.04 \%$ DMSO in artificial CSF (Baker-Herman et al., 2004).

Histology and placement verification. The local diffusion of the injected compound was confirmed after histological examination of the effect of the injection of Pontamine blue (data not shown) and BDNF or antiBDNF antibody (see Fig. $1 B$ ). The anti-BDNF antibody was injected at the beginning of the light period, and rats were killed $10 \min (n=2) ; 2 \mathrm{~h}$ $(n=2)$ (see Fig. $1 B$, left panel), or $9 \mathrm{~h}(n=1)$ after the injection. BDNF was also injected at light onset, and rats were perfused 30 min later (see Fig. $1 B$, right panel) The placement of the electrodes was verified in coronal sections stained with cresyl violet. In all cases, the deep electrode was located within layer $V$, whereas the superficial electrode was in layers 
A

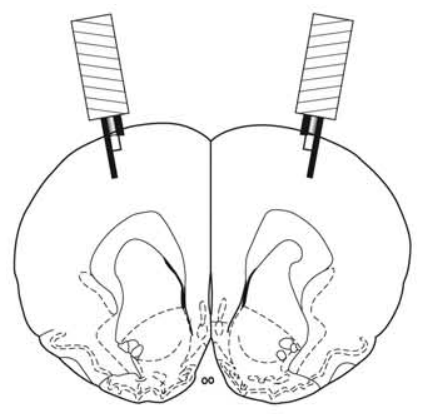

C

BDNF or control (vehicle)

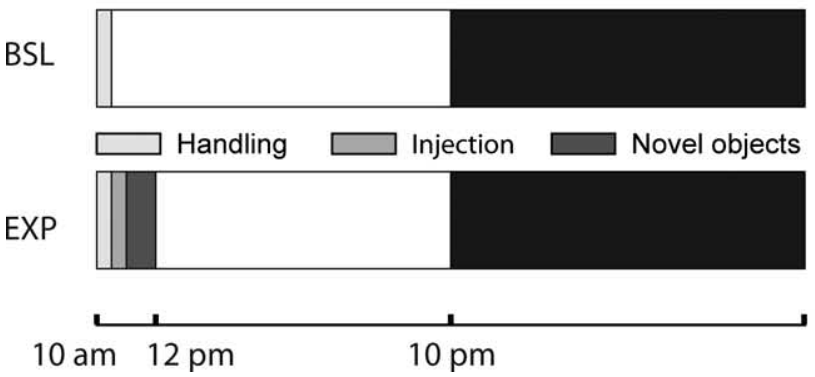

B

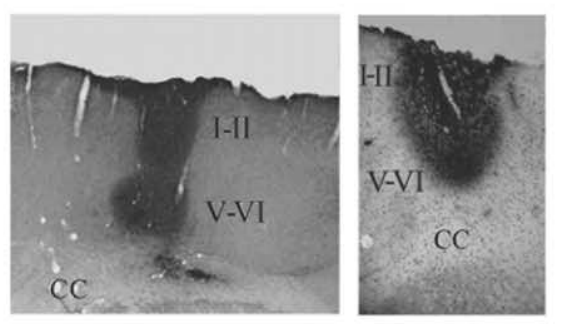

D
BDNF blockers (K252a, anti-BDNF antibody) or control (vehicle - anti-NT3 antibody)
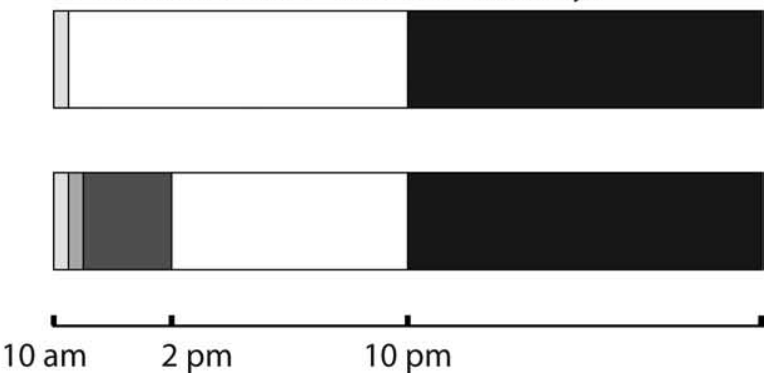

Figure 1. Experimental protocol. $A$, Schematic of a rat brain coronal section $[B,+2.70$ according to Paxinos and Watson (2005)] showing the position of the two frontal microinjection cannula/bipolar wire electrodes. $B, A B C$ staining of the diffusion of BDNF (right) and anti-BDNF (left) $\sim 30$ and 120 min after the injections, respectively. CC, Corpus callosum; I-II, cortical layers I and $\mathrm{Il}$; V-VI, cortical layers V and VI. C, Experimental design for the injection of BDNF and its vehicle. D, Experimental design for the injection of BDNF blockers and their corresponding control. BSL, Baseline day (24 $\mathrm{h}$ before the injection); EXP, experimental day. Every day the animals were gently handled for $30 \mathrm{~min}$ after light onset. The day of the injection, rats were first handled as usual, injected soon afterward, and then kept awake by exposure to novel objects for either $1 \mathrm{~h}(\boldsymbol{C})$ or $4 \mathrm{~h}(\boldsymbol{D})$. The light and dark bars indicate the light and dark period, respectively. Rats are nocturnal animals and therefore tend to sleep mostly during the day and stay awake during the night.

I-II. All animals used for the experiments showed opposite polarity in the EEG signal when superficial and deep electrodes were independently referenced to the cerebellar screw.

Statistical analysis. The statistical significance of the effects of the injections on the EEG power spectrum was assessed by Wilcoxon's nonparametric test for single frequency bins (bin size, $0.25 \mathrm{~Hz}$ ) and for frequency bands (e.g., SWA, 0.5-4 Hz). Comparisons were between the injected channel and the contralateral channel. To compute the statistical significance for the SWA time course values, a two-way repeatedmeasures ANOVA (rANOVA) with factors "time" and "derivation" (injected, contralateral) was first used, followed by paired $t$ tests (significance level adjusted for multiple comparisons by Bonferroni's correction). Differences in sleep duration and latency between groups were tested with Wilcoxon's nonparametric tests.

\section{Results}

\section{BDNF injections}

Figure $1 A$ shows a schematic of the frontal electrodes used for LFP recordings and drug delivery, and their location in the frontal cortex. Figure $1 B$ shows representative examples of local injections of BDNF or anti-BDNF. Delivery rate and injected volume were optimized in pilot experiments in which the dye Pontamine blue or a solution of $20 \%$ lidocaine were used. The latter, when injected using the final selected parameters $(0.5 \mu \mathrm{l} /$ min; $5 \mu$ l total volume), consistently and reversibly suppressed the EEG signal on the injected side, but not on the contralateral cortical area (data not shown). Figure $1 C$ summarizes the experimental protocol for the unilateral injection of BDNF and its vehicle. All injections were performed in freely moving, awake animals soon after light onset ( $\sim$ 10:30 A.M.), and in no case abnormal behavior (e.g., seizure) was observed during or after the injections. Rats were then kept awake for $1 \mathrm{~h}$ by exposure to novel objects. This time interval was selected based on a previous study in vivo that showed that most of the LTP-enhancing effect of BDNF occurs within the first 60 min after the injection (Messaoudi et al., 2002; Escobar et al., 2003). During this entire time period, no EEG abnormalities such as abnormal slow waves were visible at the site of the injection.

After the exposure to novel objects, rats were allowed to sleep ad libitum, and the effects of BDNF on their sleep were assessed. As shown in Table 1, sleep latency from the time of injection did not differ between BDNF and vehicle ( $p=0.22$, Wilcoxon's nonparametric test). Sleep architecture also was similar after all injections. Specifically, during the first $2 \mathrm{~h}$ after sleep onset, rats spent on average $84.7 \mathrm{~min}$ ( \pm 3.1 ; mean \pm SEM) in NREM sleep after BDNF and $81.5 \mathrm{~min}( \pm 3.4)$ after vehicle $(p=0.39)$ (Table $1)$. REM sleep and waking duration also did not differ between BDNF and vehicle ( $p=0.27$ and $p=0.25$, respectively).

As shown in Figure $2 A$, the day before the injection (baseline day) left and right frontal derivations showed very similar EEG power spectra during NREM sleep. In contrast, during the first $2 \mathrm{~h}$ of sleep after BDNF injection the EEG power spectrum on the injected side, compared with the contralateral side, was significantly increased between 1.5 and $4.25 \mathrm{~Hz}(p<0.05$, Wilcoxon's nonparametric test) (Fig. $2 B$ ). Computation of the entire SWA band $(0.5-4 \mathrm{~Hz})$ also revealed a significant difference between injected and contralateral frontal cortex, which lasted for $2 \mathrm{~h}$ after sleep onset (Bonferroni's post hoc, $p<0.01$, after two-way rANOVA; $\left.F_{(11,66)}=4.0382 ; p=0.00016\right)($ Fig. $2 F)$. It should be emphasized that SWA is exquisitely sensitive to the sleep/waking 
Table 1. Amount of wakefulness (W) and NREM and REM sleep after injections of BDNF, K252a, anti-BDNF, or corresponding vehicle

\begin{tabular}{|c|c|c|c|c|c|c|}
\hline & $\operatorname{BDNF}(n=7)$ & Vehicle $(n=7)$ & $\mathrm{K} 252 \mathrm{a}(n=6)$ & Vehicle $(n=6)$ & Anti-BDNF $(n=7)$ & (Vehicle) Anti-NT3 $(n=7)$ \\
\hline NREM sleep (min) & $84.66( \pm 3.13)$ & $81.5( \pm 3.44)$ & $82.79( \pm 2.46)$ & $84.09( \pm 2.54)$ & $85.19( \pm 1.46)$ & $88.48( \pm 3.02)$ \\
\hline REM sleep (min) & $11.12( \pm 1.08)$ & $10.92( \pm 2.15)$ & $11.32( \pm 1.87)$ & $14.28( \pm 1.17)$ & $12.07( \pm 1.45)$ & $16.22( \pm 1.31)$ \\
\hline $\mathrm{W}(\min )$ & $24.22( \pm 3.49)$ & $27.58( \pm 2.54)$ & $25.89( \pm 3.22)$ & $21.63( \pm 3.21)$ & $22.74( \pm 2.71)^{*}$ & $15.3( \pm 3.36)^{*}$ \\
\hline Sleep latency (min) & $76.5( \pm 3.8)$ & $81.0( \pm 4.5)$ & $202.96( \pm 8.05)$ & $210.62( \pm 5.19)$ & $197.29( \pm 7.48)$ & $198.15( \pm 8.01)$ \\
\hline
\end{tabular}

Data are presented as mean \pm SEM (in minutes) and refer to the first $2 \mathrm{~h}$ interval from sleep onset after the injections. Sleep latency is defined as the time between the start of the injection and the first consolidated sleep episode. ${ }^{*} p<0.05$, Wilcoxon's nonparametric test.
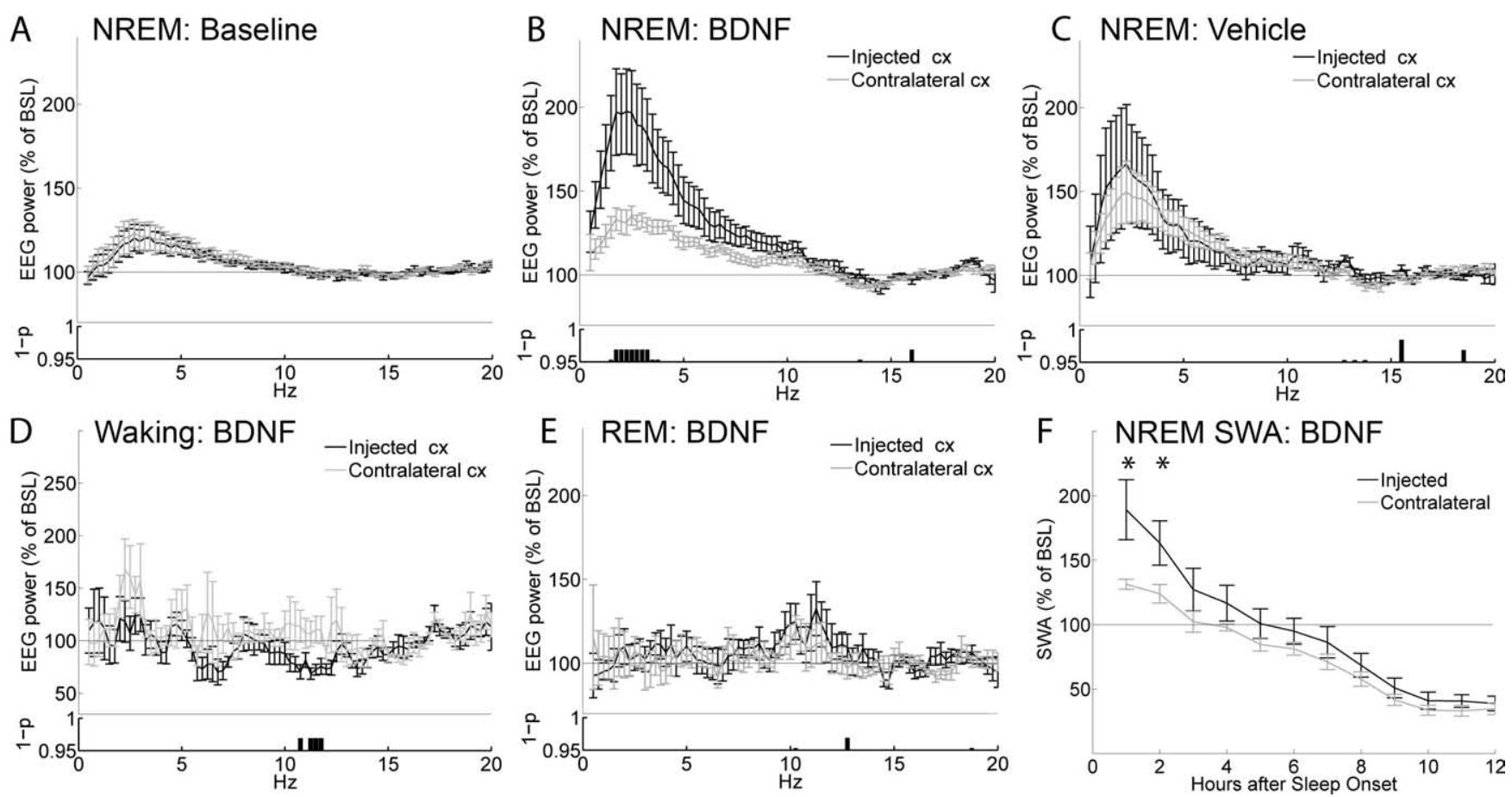

Figure 2. Effects of BDNF on the EEG power spectrum. EEG power spectrum during NREM sleep in baseline $(\boldsymbol{A})$ and after injection of BDNF $(\boldsymbol{B})$ or vehicle $(\boldsymbol{C})$. All data are mean $\pm \mathrm{SEM}$ for the first $2 \mathrm{~h}$ after sleep onset. For each frequency bin $(0.25 \mathrm{~Hz})$, values are expressed as percentage of the NREM EEG power spectrum during the first $6 \mathrm{~h}$ of baseline (BSL), normalized for the mean power density in the $15-20 \mathrm{~Hz}$ range within each interval. The bottom panels show the statistical significance for each frequency bin, comparing the injected with the contralateral hemisphere $(p<0.05$, Wilcoxon's nonparametric test). Note that the higher EEG power in the low frequencies after vehicle injections relative to baseline is expected, because rats were awake longer. $\boldsymbol{D}$, EEG power spectrum during waking over the first $3 \mathrm{~h}$ after the injection of BDNF. $E$, EEG power spectrum during REM sleep during the first $2 \mathrm{~h}$ of sleep after the injection of BDNF. $F$, Time course of the changes in SWA $\left(0.5-4 \mathrm{~Hz}\right.$ ) during NREM sleep after the injection of BDNF (Bonferroni's post hoc between injected and contralateral hemispheres, ${ }^{*} p<0.01$, for the first two $1 \mathrm{~h}$ intervals, after two-way rANOVA; $F_{(11,66)}=3.37 ; p=0.000098$ ). SWA is expressed as the mean value of relative NREM EEG power across frequencies ranging from 0.5 through $4 \mathrm{~Hz}$. Data in all panels refer to $n=7$ rats.

history of the previous 3-6 h. Thus, because waking time/distribution after light onset differed between baseline and injection day (Fig. 1), the effects of BDNF (and its blockers) (see below) on the EEG power spectrum could only be compared across the two hemispheres (injected vs contralateral), but not across days (baseline vs injection day).

The effects of BDNF on the EEG spectrum were specific for NREM sleep, because no difference between left and right frontal derivations was evident during REM sleep (Fig. 2E). Similarly, the analysis of the EEG power spectrum during waking confirmed that, after BDNF injection, there was neither an increase in SWA relative to baseline, nor SWA asymmetries between injected and contralateral side. A selective increase in the waking EEG power between 11.5 and $12.5 \mathrm{~Hz}$ was instead present in the injected site compared with the contralateral derivation (Fig. $2 D$ ). Finally, after vehicle injection, no significant difference in any frequency band of the NREM EEG power spectrum was detected between injected and contralateral frontal cortex (Fig. 2C). Because of practical limitations in the number of animals to be implanted for local field potential recordings and local injections, the effects of BDNF could not be directly compared with enough statistical power to those of its vehicle. This is again attributable to the high sensitivity of SWA to the sleep/waking history of the previous 3-6 h: rats remained awake for $\sim 80$ min after both BDNF and vehicle injections (Table 1), but their sleep/waking history for the $2-3 \mathrm{~h}$ preceding the injections varied significantly.

In summary, local BDNF injections can specifically and reversibly dissociate the intensity of sleep between the two hemispheres, as measured by the NREM EEG power spectrum within the SWA band, without affecting the pattern and duration of sleep.

\section{K252a injections}

We next tested whether the cortical injection during waking of K252a, a blocker of the BDNF receptor TrkB, could blunt the subsequent SWA response during sleep. Figure $1 D$ shows that, as for BDNF, K252a (and the corresponding vehicle) was injected in the frontal cortex soon after light onset. In contrast to the BDNF injections, however, rats injected with $\mathrm{K} 252 \mathrm{a}$ and its vehicle were kept awake longer, for $\sim 4 \mathrm{~h}$, to allow a significant buildup of sleep pressure.

No abnormal behavior was observed during or after the injec- 

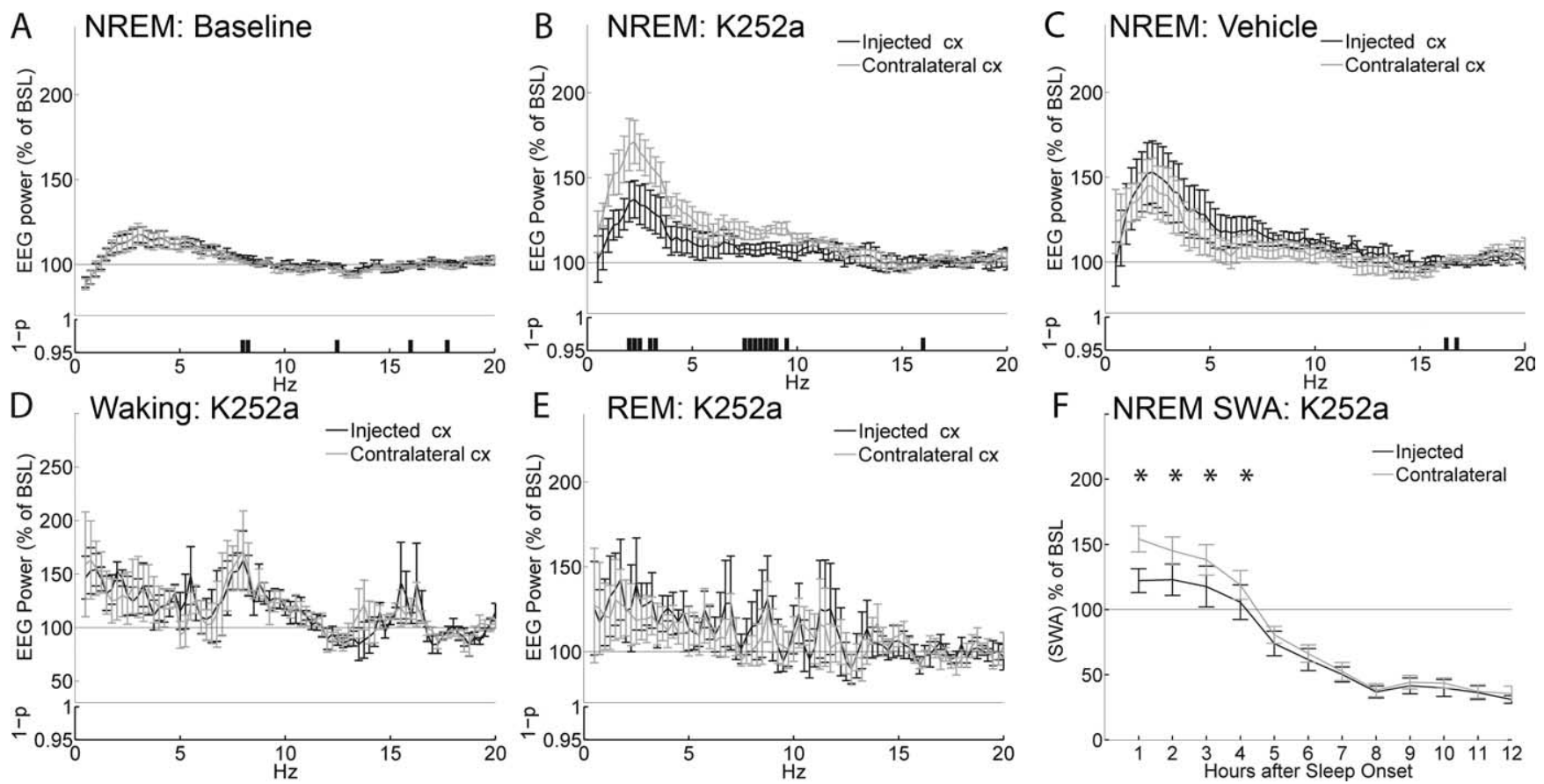

Figure 3. Effects of K252a on the EEG power spectrum. EEG power spectrum during NREM sleep in baseline $(\boldsymbol{A})$ and after injection of K252a (B) or vehicle $(\boldsymbol{C}$. All data are mean \pm SEM for the first $2 \mathrm{~h}$ after sleep onset. For each frequency bin $(0.25 \mathrm{~Hz})$, values are expressed as percentage of the NREM EEG power spectrum during the first $6 \mathrm{~h}$ of baseline (BSL), normalized for the mean power density in the $15-20 \mathrm{~Hz}$ range within each interval. The bottom panels show the statistical significance for each frequency bin, comparing the injected with the contralateral hemisphere $(p<0.05$, Wilcoxon's nonparametric test). Note that the higher EEG power in the low frequencies after vehicle injections relative to baseline is expected, because rats were awake longer. $\boldsymbol{D}$, EEG power spectrum during waking for the first $3 \mathrm{~h}$ after the injection of K252a. $\boldsymbol{E}$, EEG power spectrum during REM sleep during the first $2 \mathrm{~h}$ of sleep after injection of K252a. $\boldsymbol{F}$, Time course of the changes in SWA $\left(0.5-4 \mathrm{~Hz}\right.$ ) during NREM sleep after the injection of K252a (Bonferroni's post hoc between injected and contralateral hemispheres, ${ }^{*} p<0.01$, for the first four $1 \mathrm{~h}$ intervals, after two-way rANOVA; $\left.F_{(11,55)}=11.79 ; p<0.00001\right)$. SWA is expressed as the mean value of relative normalized NREM EEG power across frequencies ranging from 0.5 through $4 \mathrm{~Hz}$. Data in all panels refer to $n=6$ rats.

tion of K252a. Table 1 shows that sleep latency from the time of injection was $203.0 \mathrm{~min}( \pm 8.1)$ after K252a and $210.6 \mathrm{~min}( \pm 5.2)$ after vehicle ( $p=0.22$, Wilcoxon's nonparametric test). Moreover, during the first $2 \mathrm{~h}$ after sleep onset, rats spent a similar amount of time in NREM sleep after K252a and vehicle ( $p=$ 0.49 ) (Table 1). No significant difference between K252a and vehicle was found for REM sleep duration and time spent awake.

As in the previous experiments with BDNF, left and right frontal derivations showed very similar EEG power spectra in NREM sleep during baseline (i.e., the day before K252a was injected) (Fig. 3A). After the injection, as expected, $4 \mathrm{~h}$ of continuous waking led to a robust SWA increase above baseline levels in the noninjected frontal derivation during the first $2 \mathrm{~h}$ after sleep onset. The side injected with K252a, however, compared with the contralateral side, showed a decrease in NREM EEG power in two clusters of frequencies, one within the SWA band $(2-3.5 \mathrm{~Hz})$ and the other between 7.5 and $9.5 \mathrm{~Hz}$ (Fig. 3B). Computation of the entire SWA band also revealed a significant difference between injected and contralateral frontal cortex that persisted for $4 \mathrm{~h}$ after sleep onset (Fig. $3 F$ ). No difference between right and left hemispheres was detected in waking (Fig. 3D) or REM sleep (Fig. $3 E$ ), indicating that the effects were specific for NREM sleep. Finally, no asymmetry in the EEG power spectrum of NREM sleep was detected after vehicle injections (Fig. $3 C$ ). Thus, local K252a injections can decrease the EEG power spectrum during NREM sleep, including SWA, without affecting the pattern and duration of sleep.

\section{Anti-BDNF injections}

Finally, we tested whether an anti-BDNF antibody delivered in the cortex during waking could also attenuate the subsequent
SWA response during sleep. To better control for aspecific effects, the results of anti-BDNF injections were compared with those of injections of an antibody against another neurotrophin, NT3. In pilot experiments, the anti-BDNF antibody was injected at the beginning of the light period, and rats were killed $10 \mathrm{~min}$, and 2, 4 , or $9 \mathrm{~h}$ after the injection. After $\mathrm{ABC}$ staining, we found that the antibody diffusion was confined to the injected site (Fig. $1 B$ ) and was almost completely undetectable after $9 \mathrm{~h}$ from the time of injection (data not shown). As for the previous experiments, left and right frontal derivations showed very similar EEG power spectra in NREM sleep during baseline (Fig. 4A). Also, as before, the anti-BDNF antibody was injected in the frontal cortex soon after light onset, and no abnormal behavior was observed afterward. As for the experiments with K252a, rats were kept awake for $\sim 4$ h (Fig. 1D), which resulted in a significant SWA increase above baseline levels in the noninjected frontal derivation (Fig. $4 B$ ). The EEG power between 0.5 and $4.25 \mathrm{~Hz}$, however, was significantly and specifically reduced on the injected side relative to the contralateral cortex (Fig. 4B). The blunted SWA response after anti-BDNF injections lasted for $\sim 4 \mathrm{~h}$ (Fig. $4 F$ ), and no differences in the EEG power during REM sleep (Fig. $4 E$ ) were detected between right and left hemispheres, again indicating that the effects were specific for NREM sleep. During the first $3 \mathrm{~h}$ of waking after the injection, a cluster of frequencies between 3.5 and $5.25 \mathrm{~Hz}$ was significantly different in the injected side compared with the contralateral side (Fig. $4 D$ ). Finally, no asymmetry in NREM EEG power was detected after injections of anti-NT3 (Fig. 4C).

Table 1 shows that rats were awake $\sim 7$ min longer after antiBDNF injections than after anti-NT3 injections $(22.7 \pm 1.0$ vs $15.3 \pm 1.3 \mathrm{~min} ; p=0.042$, paired $t$ test). The increase in waking 

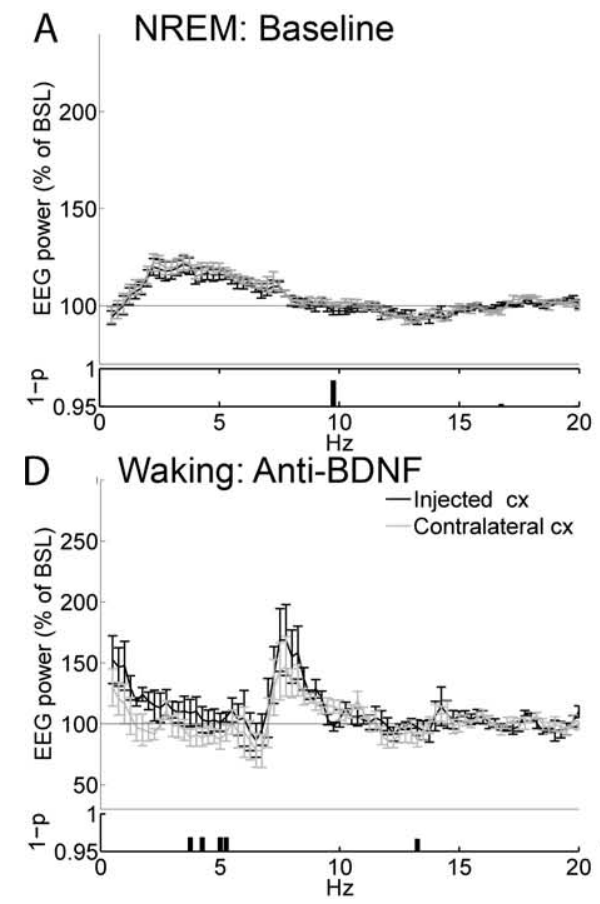
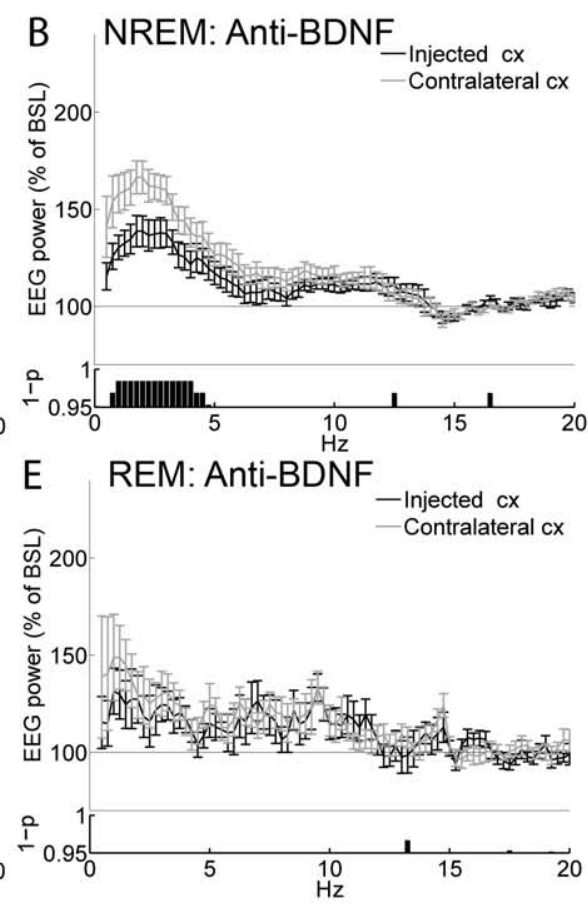

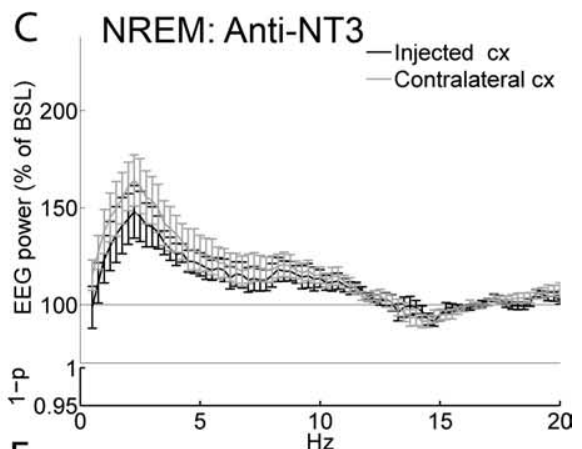

$\mathrm{F}$

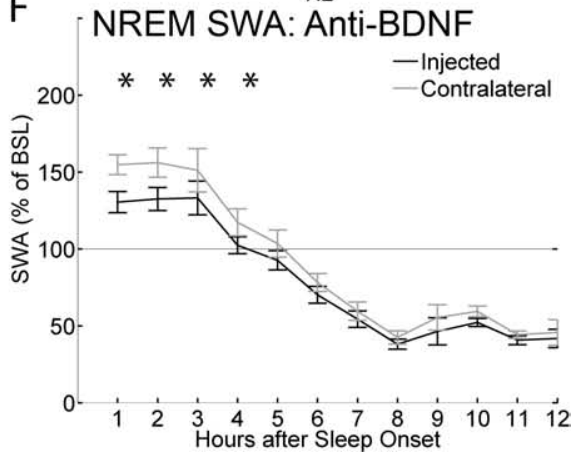

Figure 4. Effects of the anti-BDNF antibody on the EEG power spectrum. EEG power spectrum during NREM sleep in baseline $(\boldsymbol{A})$ and after injection of anti-BDNF $(\boldsymbol{B})$ or anti-NT3 $(\boldsymbol{C})$. All data are mean \pm SEM for the first $2 \mathrm{~h}$ after sleep onset. For each frequency bin $(0.25 \mathrm{~Hz})$, values are expressed as percentage of the NREM EEG power spectrum during the first $6 \mathrm{~h}$ of baseline (BSL), normalized for the mean power density in the $15-20 \mathrm{~Hz}$ range within each interval. The bottom panels show the statistical significance for each frequency bin, comparing the injected with the contralateral hemisphere ( $p<0.05$, Wilcoxon's nonparametric test). Note that the higher EEG power in the low frequencies after vehicle injections relative to baseline is expected, because rats were awake longer. $\boldsymbol{D}$, EEG power spectrum during waking for the first $3 \mathrm{~h}$ after the injection of anti-BDNF. $E$, EEG power spectrum during REM sleep during the first $2 \mathrm{~h}$ of sleep after the injection of anti-BDNF. $\boldsymbol{F}$, Time course of the changes in NREM SWA after the injection of anti-BDNF (Bonferroni's post hoc between injected and contralateral hemispheres, ${ }^{*} p<0.01$, for the first four $1 \mathrm{~h}$ intervals after two-way rANOVA; $\left.F_{(11,66)}=7.3 ; p<0.00001\right)$. SWA is expressed as the mean value of relative normalized NREM EEG power across frequencies ranging from 0.5 through $4 \mathrm{~Hz}$. Data in all panels refer to $n=7$ rats.

was mostly at the expense of REM sleep ( $p=0.08$, paired $t$ test), whereas NREM sleep duration and sleep latency did not differ between anti-BDNF and anti-NT3 injections ( $p=0.5$, Wilcoxon's nonparametric test). Thus, local anti-BDNF injections blunt the SWA homeostatic response normally observed after prolonged waking, but have little or no effects on other sleep parameters.

\section{Discussion}

SWA is considered a reliable EEG marker of NREM sleep intensity and sleep need, but the mechanisms underlying its homeostatic regulation as a function of previous waking remain unclear. On one hand, the homeostatic SWA increase could simply be an epiphenomenon without physiological significance. On the other hand, SWA could reflect, at the EEG level, the cellular processes that trigger the need for sleep after prolonged waking. Recent studies showing that SWA can be homeostatically regulated at a local level support the latter possibility. In rodents, unilateral whisker stimulation results in an increase in SWA in the contralateral hemisphere (Vyazovskiy et al., 2000, 2004). In humans, a mechanical vibration applied for several hours to one hand leads to a selective increase of sleep SWA in the stimulated hemisphere (Kattler et al., 1994). Other studies indicate that SWA reflects not just previous "use" of specific neuronal circuits, but rather the occurrence of plastic changes. Visuomotor adaptation in humans resulted in a local SWA increase only in the cortical areas specifically involved in learning the task (Huber et al., 2004). Because this study controlled for the effects of motor activity, SWA changes could be attributed to the occurrence of plastic changes rather than to the use of specific motor circuits. In a second study, high-frequency transcranial magnetic stimulation
(TMS) over motor cortex led to a local increase in the amplitude of EEG responses to TMS pulses, indicative of potentiation of premotor circuits. This was followed by a local increase in sleep SWA. Moreover, the magnitude of potentiation during wakefulness predicted the local SWA increase during sleep (Huber et al., 2007b). Together, these experiments suggest that sleep SWA is affected by plastic changes in local cortical circuits and, more specifically, that SWA should increase with synaptic potentiation.

Additional evidence for a connection between synaptic plasticity and SWA regulation comes from a study in rats in which, for the same duration of wakefulness, the amount of SWA during subsequent sleep was positively correlated with the amount of exploration of an enriched environment (Huber et al., 2007a). Intriguingly, exploratory behavior was also correlated with the cortical expression of $B D N F$, and the latter predicted the increase in sleep SWA. Based on this observation, we hypothesized that the induction of BDNF in the cortex could represent a causal link between exploratory behavior and the homeostatic regulation of sleep SWA. Evidence in favor of this hypothesis also comes from the observation that the increase in SWA after sleep deprivation only occurs in rats after postnatal day 20, precisely the time at which sleep deprivation begins to induce cortical BDNF expression (Hairston et al., 2004).

The results of the present experiments strongly support the postulated causal relationship between BDNF and SWA regulation. First, the local infusion of BDNF in the rat frontal cerebral cortex during waking was followed by a significant increase in sleep SWA in the injected side relative to the contralateral, noninjected area. The increase was specific for NREM sleep, and 
reversible within $2 \mathrm{~h}$ from sleep onset, suggesting that it was not attributable to aspecific effects of the infusion or to a pathological slowing of the EEG. Second, the infusion of two different BDNF blockers during enforced wakefulness resulted in a blunted SWA response in the injected frontal cortex relative to the contralateral side. Again, this effect was specific for NREM sleep, and reversible within $4 \mathrm{~h}$ from sleep onset. This finding indicates that the endogenous induction of BDNF is involved in triggering the homeostatic SWA response that is observed after both spontaneous wakefulness and sleep deprivation. Consistent with this view, $B D N F$ mRNA levels are higher after wakefulness than after sleep, and increase further after sleep deprivation (Cirelli and Tononi, 2000). Third, both the increase in SWA after BDNF and its decrease after BDNF blockers occurred with no or minimal changes in sleep amount, suggesting that cortical BDNF can locally affect sleep intensity independently of sleep duration. Concerning this last observation, a previous study found that intracerebroventricular injections of BDNF at high doses increased the time spent in NREM sleep in rats and rabbits without affecting SWA (Kushikata et al., 1999). However, unlike intracortical injections, intracerebroventricular injections can affect sleep duration by acting on subcortical structures, such as the hypothalamus and the basal forebrain, which are involved in the central control of sleep duration (Jones, 2005).

What are the mechanisms by which BDNF leads to a subsequent increase in sleep SWA? The most straightforward possibility is that BDNF may do so by promoting synaptic potentiation in local circuits. In preparation for the present study, we attempted to measure cortical evoked responses after transcallosal stimulation from an area close to the site of the injection, to compare postinjection changes in slope and amplitude relative to the contralateral, noninjected area. However, injections in close proximity to the recording electrode interfered with the recording of stable and reliable responses. Although we could not show that BDNF injection induced synaptic potentiation in the same animals in which we observed an increase in SWA, there is compelling previous evidence that BDNF is involved in synaptic potentiation. At Schaffer collateral-CA1 synapses in hippocampal slices, BDNF application produces a dramatic and sustained enhancement of synaptic strength (Kang and Schuman, 1995). Also, hippocampal slices from BDNF knock-out animals show impaired LTP induction that can be restored by reintroducing BDNF (Korte et al., 1995; Patterson et al., 1996), and BDNF promotes the cytoskeletal changes that support LTP in the adult brain (Rex et al., 2007). Moreover, in rat hippocampal slices, short high-frequency bursts of electrical stimuli able to induce LTP also upregulate BDNF expression (Patterson et al., 1992) and trigger its secretion (Gooney and Lynch, 2001; Gartner and Staiger, 2002). In the hippocampus in vivo, BNDF is rapidly and selectively induced after contextual learning (Hall et al., 2000). BDNF expression increases in prefrontal areas after learning an odor discrimination task (Naimark et al., 2007), and in motor areas after motor training (Klintsova et al., 2004). Finally, in vivo exogenous application of BDNF is sufficient to induce a longterm increase in synaptic strength that parallels many features of late-phase LTP (Messaoudi et al., 1998, 2002, 2007; Ying et al., 2002). Importantly, synaptic potentiation saturates $\sim 1 \mathrm{~h}$ after the infusion (Messaoudi et al., 2002), which led to our choice to keep animals awake for $1 \mathrm{~h}$ before measuring the effects on SWA.

Other mechanisms in addition to synaptic potentiation, not necessarily mutually exclusive, may also be involved. BDNF could affect SWA through its positive action on the local release of acetylcholine and/or glutamate (Knipper et al., 1994; Sala et al.,
1998), or through its effects leading to a reduction of GABAergic inhibition (Wardle and Poo, 2003; Sale et al., 2007). However, recent evidence obtained in freely moving rats suggests that the release of these neurotransmitters or neuromodulators per se may not be sufficient to affect SWA, unless associated with active (presumably exploratory) waking. Specifically, it was found that the stimulation of the cholinergic basal forebrain by local infusion of the glutamatergic agonist NMDA resulted in an increase in wakefulness with high theta activity, as well as an increase in the subsequent SWA response (Wigren et al., 2007). In contrast, the infusion of another glutamatergic agonist, AMPA, which did not increase active, theta-rich, waking, did not increase SWA (Wigren et al., 2007). Because naturalistic theta-burst stimulation causes the release of BDNF (Balkowiec and Katz, 2002; Aicardi et al., 2004) and the perfusion of BDNF blockers before or during the same stimulation precludes the induction of LTP (Figurov et al., 1996; Chen et al., 1999), these data are consistent with the idea that waking-associated synaptic potentiation, rather than simply neuronal activation, may be linked to sleep homeostasis.

If BDNF, whether exogenously applied or released during exploratory activities, leads to synaptic potentiation, how would such potentiation lead in turn to an increase in SWA? A likely scenario is provided by large-scale computer simulations of corticothalamic circuits under conditions of low and high synaptic strength (Esser et al., 2007). These simulations show that a local increase in the strength of corticocortical connections powerfully affects the dynamics of cortical slow oscillations, the alternation between depolarized up states and hyperpolarized down states at $\sim 1 \mathrm{~Hz}$ that underlies sleep SWA (Steriade et al., 2001). In these simulations, a local increase in synaptic strength led, through an increase in the size of EPSPs, to a larger amplitude of single-cell slow oscillations. Moreover, the increased coupling among connected cells led to a stronger synchronization of single-unit slow oscillations across the network. These two effects combined led to slow waves of larger amplitude in simulated local recordings, and ultimately to an increase in power in the estimated SWA band.

\section{References}

Achermann P, Borbely AA (2003) Mathematical models of sleep regulation. Front Biosci 8:s683-s693.

Aicardi G, Argilli E, Cappello S, Santi S, Riccio M, Thoenen H, Canossa M (2004) Induction of long-term potentiation and depression is reflected by corresponding changes in secretion of endogenous brain-derived neurotrophic factor. Proc Natl Acad Sci USA 101:15788-15792.

Akaneya Y, Tsumoto T, Kinoshita S, Hatanaka H (1997) Brain-derived neurotrophic factor enhances long-term potentiation in rat visual cortex. J Neurosci 17:6707-6716.

Amzica F, Steriade M (1995) Disconnection of intracortical synaptic linkages disrupts synchronization of a slow oscillation. J Neurosci 15:4658-4677.

Baker-Herman TL, Fuller DD, Bavis RW, Zabka AG, Golder FJ, Doperalski NJ, Johnson RA, Watters JJ, Mitchell GS (2004) BDNF is necessary and sufficient for spinal respiratory plasticity following intermittent hypoxia. Nat Neurosci 7:48-55.

Balkowiec A, Katz DM (2002) Cellular mechanisms regulating activitydependent release of native brain-derived neurotrophic factor from hippocampal neurons. J Neurosci 22:10399-10407.

Borbely AA (1982) A two process model of sleep regulation. Hum Neurobiol 1:195-204.

Caleo M, Menna E, Chierzi S, Cenni MC, Maffei L (2000) Brain-derived neurotrophic factor is an anterograde survival factor in the rat visual system. Curr Biol 10:1155-1161.

Chen G, Kolbeck R, Barde YA, Bonhoeffer T, Kossel A (1999) Relative contribution of endogenous neurotrophins in hippocampal long-term potentiation. J Neurosci 19:7983-7990.

Cirelli C, Tononi G (2000) Differential expression of plasticity-related genes 
in waking and sleep and their regulation by the noradrenergic system. J Neurosci 20:9187-9194.

Escobar ML, Figueroa-Guzman Y, Gomez-Palacio-Schjetnan A (2003) In vivo insular cortex LTP induced by brain-derived neurotrophic factor. Brain Res 991:274-279.

Esser SK, Hill SL, Tononi G (2007) Sleep homeostasis and cortical synchronization: I. Modeling the effects of synaptic strength on sleep slow waves. Sleep 30:1617-1630.

Figurov A, Pozzo-Miller LD, Olafsson P, Wang T, Lu B (1996) Regulation of synaptic responses to high-frequency stimulation and LTP by neurotrophins in the hippocampus. Nature 381:706-709.

Gartner A, Staiger V (2002) Neurotrophin secretion from hippocampal neurons evoked by long-term-potentiation-inducing electrical stimulation patterns. Proc Natl Acad Sci USA 99:6386-6391.

Gooney M, Lynch MA (2001) Long-term potentiation in the dentate gyrus of the rat hippocampus is accompanied by brain-derived neurotrophic factor-induced activation of TrkB. J Neurochem 77:1198-1207.

Hairston IS, Peyron C, Denning DP, Ruby NF, Flores J, Sapolsky RM, Heller HC, O'Hara BF (2004) Sleep deprivation effects on growth factor expression in neonatal rats: a potential role for BDNF in the mediation of delta power. J Neurophysiol 91:1586-1595.

Hall J, Thomas KL, Everitt BJ (2000) Rapid and selective induction of BDNF expression in the hippocampus during contextual learning. Nat Neurosci 3:533-535.

Huber R, Ghilardi MF, Massimini M, Tononi G (2004) Local sleep and learning. Nature 430:78-81.

Huber R, Ghilardi MF, Massimini M, Ferrarelli F, Riedner BA, Peterson MJ, Tononi G (2006) Arm immobilization causes cortical plastic changes and locally decreases sleep slow wave activity. Nat Neurosci 9:1169-1176.

Huber R, Tononi G, Cirelli C (2007a) Exploratory behavior, cortical BDNF expression, and sleep homeostasis. Sleep 30:129-139.

Huber R, Esser SK, Ferrarelli F, Massimini M, Peterson MJ, Tononi G (2007b) TMS-induced cortical potentiation during wakefulness locally increases slow wave activity during sleep. PLoS ONE 2:e276.

Jha SK, Jones BE, Coleman T, Steinmetz N, Law CT, Griffin G, Hawk J, Dabbish N, Kalatsky VA, Frank MG (2005) Sleep-dependent plasticity requires cortical activity. J Neurosci 25:9266-9274.

Jiang B, Akaneya Y, Hata Y, Tsumoto T (2003) Long-term depression is not induced by low-frequency stimulation in rat visual cortex in vivo: a possible preventing role of endogenous brain-derived neurotrophic factor. J Neurosci 23:3761-3770.

Jones BE (2005) From waking to sleeping: neuronal and chemical substrates. Trends Pharmacol Sci 26:578-586.

Kang H, Schuman EM (1995) Long-lasting neurotrophin-induced enhancement of synaptic transmission in the adult hippocampus. Science 267:1658-1662.

Kattler H, Dijk DJ, Borbely AA (1994) Effect of unilateral somatosensory stimulation prior to sleep on the sleep EEG in humans. J Sleep Res 3:159-164

Klintsova AY, Dickson E, Yoshida R, Greenough WT (2004) Altered expression of BDNF and its high-affinity receptor TrkB in response to complex motor learning and moderate exercise. Brain Res 1028:92-104.

Knipper M, da Penha Berzaghi M, Blochl A, Breer H, Thoenen H, Lindholm D (1994) Positive feedback between acetylcholine and the neurotrophins nerve growth factor and brain-derived neurotrophic factor in the rat hippocampus. Eur J Neurosci 6:668-671.

Korte M, Carroll P, Wolf E, Brem G, Thoenen H, Bonhoeffer T (1995) Hippocampal long-term potentiation is impaired in mice lacking brainderived neurotrophic factor. Proc Natl Acad Sci USA 92:8856-8860.

Kossel AH, Cambridge SB, Wagner U, Bonhoeffer T (2001) A caged Ab reveals an immediate/instructive effect of BDNF during hippocampal synaptic potentiation. Proc Natl Acad Sci USA 98:14702-14707.

Kushikata T, Fang J, Krueger JM (1999) Brain-derived neurotrophic factor enhances spontaneous sleep in rats and rabbits. Am J Physiol 276:R1334-R1338.

Lu B (2003) BDNF and activity-dependent synaptic modulation. Learn Mem 10:86-98.

Martinez-Gonzalez D, Lesku JA, Rattenborg NC (2008) Increased EEG spectral power density during sleep following short-term sleep deprivation in pigeons (Columba livia): evidence for avian sleep homeostasis. J Sleep Res, in press.

Messaoudi E, Bardsen K, Srebro B, Bramham CR (1998) Acute intrahippocampal infusion of BDNF induces lasting potentiation of synaptic transmission in the rat dentate gyrus. J Neurophysiol 79:496-499.

Messaoudi E, Ying SW, Kanhema T, Croll SD, Bramham CR (2002) Brainderived neurotrophic factor triggers transcription-dependent, late phase long-term potentiation in vivo. J Neurosci 22:7453-7461.

Messaoudi E, Kanhema T, Soule J, Tiron A, Dagyte G, da Silva B, Bramham CR (2007) Sustained Arc/Arg3.1 synthesis controls long-term potentiation consolidation through regulation of local actin polymerization in the dentate gyrus in vivo. J Neurosci 27:10445-10455.

Nagappan G, Lu B (2005) Activity-dependent modulation of the BDNF receptor TrkB: mechanisms and implications. Trends Neurosci 28:464-471.

Naimark A, Barkai E, Matar MA, Kaplan Z, Kozlovsky N, Cohen H (2007) Upregulation of neurotrophic factors selectively in frontal cortex in response to olfactory discrimination learning. Neural Plast 2007:13427.

Patterson SL, Grover LM, Schwartzkroin PA, Bothwell M (1992) Neurotrophin expression in rat hippocampal slices: a stimulus paradigm inducing LTP in CA1 evokes increases in BDNF and NT-3 mRNAs. Neuron 9:1081-1088.

Patterson SL, Abel T, Deuel TA, Martin KC, Rose JC, Kandel ER (1996) Recombinant BDNF rescues deficits in basal synaptic transmission and hippocampal LTP in BDNF knockout mice. Neuron 16:1137-1145.

Paxinos G, Watson C (2005) The rat brain in stereotaxic coordinates, Ed 5. Amsterdam: Elsevier Academic.

Rex CS, Lin CY, Kramar EA, Chen LY, Gall CM, Lynch G (2007) Brainderived neurotrophic factor promotes long-term potentiation-related cytoskeletal changes in adult hippocampus. J Neurosci 27:3017-3029.

Sala R, Viegi A, Rossi FM, Pizzorusso T, Bonanno G, Raiteri M, Maffei L (1998) Nerve growth factor and brain-derived neurotrophic factor increase neurotransmitter release in the rat visual cortex. Eur J Neurosci 10:2185-2191.

Sale A, Maya Vetencourt JF, Medini P, Cenni MC, Baroncelli L, De Pasquale R, Maffei L (2007) Environmental enrichment in adulthood promotes amblyopia recovery through a reduction of intracortical inhibition. Nat Neurosci 10:679-681.

Steriade M, Timofeev I, Grenier F (2001) Natural waking and sleep states: a view from inside neocortical neurons. J Neurophysiol 85:1969-1985.

Thoenen H (2000) Neurotrophins and activity-dependent plasticity. Prog Brain Res 128:183-191.

Tobler I (2005) Phylogeny of sleep regulation. In: Principles and practice of sleep medicine (Kryger MH, Roth T, Dement WC, eds), pp 77-90. Philadelphia: Elsevier.

Tobler I, Franken P, Gao B, Jaggi K, Borbely AA (1994) Sleep deprivation in the rat at different ambient temperatures: effect on sleep, EEG spectra and brain temperature. Arch Ital Biol 132:39-52.

Vyazovskiy V, Borbely AA, Tobler I (2000) Unilateral vibrissae stimulation during waking induces interhemispheric EEG asymmetry during subsequent sleep in the rat. J Sleep Res 9:367-371.

Vyazovskiy VV, Welker E, Fritschy JM, Tobler I (2004) Regional pattern of metabolic activation is reflected in the sleep EEG after sleep deprivation combined with unilateral whisker stimulation in mice. Eur J Neurosci 20:1363-1370.

Wardle RA, Poo MM (2003) Brain-derived neurotrophic factor modulation of GABAergic synapses by postsynaptic regulation of chloride transport. J Neurosci 23:8722-8732.

Wigren HK, Schepens M, Matto V, Stenberg D, Porkka-Heiskanen T (2007) Glutamatergic stimulation of the basal forebrain elevates extracellular adenosine and increases the subsequent sleep. Neuroscience 147:811-823.

Ying SW, Futter M, Rosenblum K, Webber MJ, Hunt SP, Bliss TV, Bramham CR (2002) Brain-derived neurotrophic factor induces long-term potentiation in intact adult hippocampus: requirement for ERK activation coupled to CREB and upregulation of Arc synthesis. J Neurosci 22:15321540. 